\title{
Coherence tomography with broad bandwidth extreme ultraviolet and soft X-ray radiation
}

\author{
S. Skruszewicz ${ }^{1,2}$ (D) S. Fuchs ${ }^{1,2} \cdot$ J. J. Abel ${ }^{1,2} \cdot$ J. Nathanael ${ }^{1,2} \cdot$ J. Reinhard ${ }^{1,2} \cdot$ C. Rödel ${ }^{1,2} \cdot$ F. Wiesner $^{1,2}$. \\ M. Wünsche ${ }^{1,2} \cdot$ P. Wachulak ${ }^{3} \cdot$ A. Bartnik ${ }^{3} \cdot$ K. Janulewicz ${ }^{3} \cdot$ H. Fiedorowicz ${ }^{3} \cdot$ G. G. Paulus ${ }^{1,2}$
}

Received: 12 June 2020 / Accepted: 4 February 2021 / Published online: 24 March 2021

(c) The Author(s) 2021

\begin{abstract}
We present an overview of recent results on optical coherence tomography with the use of extreme ultraviolet and soft X-ray radiation (XCT). XCT is a cross-sectional imaging method that has emerged as a derivative of optical coherence tomography (OCT). In contrast to OCT, which typically uses near-infrared light, XCT utilizes broad bandwidth extreme ultraviolet (XUV) and soft X-ray (SXR) radiation (Fuchs et al in Sci Rep 6:20658, 2016). As in OCT, XCT's axial resolution only scales with the coherence length of the light source. Thus, an axial resolution down to the nanometer range can be achieved. This is an improvement of up to three orders of magnitude in comparison to OCT. XCT measures the reflected spectrum in a commonpath interferometric setup to retrieve the axial structure of nanometer-sized samples. The technique has been demonstrated with broad bandwidth XUV/SXR radiation from synchrotron facilities and recently with compact laboratory-based laserdriven sources. Axial resolutions down to $2.2 \mathrm{~nm}$ have been achieved experimentally. XCT has potential applications in three-dimensional imaging of silicon-based semiconductors, lithography masks, and layered structures like XUV mirrors and solar cells.
\end{abstract}

\section{Introduction}

Optical Coherence Tomography (OCT) is a well-established technique providing cross-sectional imaging of samples in a non-invasive and contact-free manner using infrared and visible light $[1,2]$. Since its invention in the 90 s $[3,4]$, it has become a standard diagnostic technique in ophthalmology, especially for investigating diseases of the retina in vivo $[5,6]$. Meanwhile, the capabilities of OCT have also been employed in industrial non-destructive testing [7-9] and preservation of art heritage objects [10-13] and in the field of archeology [14].

OCT is based on low coherence interferometry $[4,15]$. The time delay and the intensity of backscattered light from the sample are measured by evaluating the interference of

S. Skruszewicz

slawomir.skruszewicz@desy.de

1 Institute of Optics and Quantum Electronics, Friedrich Schiller University Jena, Max-Wien-Platz 1, 07743 Jena, Germany

2 Helmholtz Institute Jena, Fröbelstieg 3, 07743 Jena, Germany

3 Institute of Optoelectronics, Military University of Technology, 2 Kaliskiego Str., 00-980 Warsaw, Poland a light beam with low temporal coherence from a reference arm and the sample arm. Technically, the experiment is realized by splitting an illumination beam into a reference and a signal beam using, e.g., a Michelson-type interferometer, where one of the mirrors is replaced by the sample. The rescattered light from the sample is superimposed with the light from the reference arm. Due to a broad bandwidth of the illumination and as a consequence of its short coherence length, interference only occurs if the path difference of the two beams is smaller than the coherence length. Thus, by scanning the path difference, the axial structure of the sample in the direction of the optical axis can be probed (time-domain OCT). Alternatively, the axial information can be obtained for a fixed path-difference by analyzing the spectrum of the backscattered light at the output of the interferometer (frequency-domain OCT). Additionally, via conventional imaging, the lateral structure of the sample can be obtained by scanning a focused illumination beam across the sample.

One of the main advantages of OCT is that the axial and the lateral resolutions are decoupled. The lateral resolution is limited by the focus spot size provided by the conventional imaging, whereas the axial resolution is equivalent to the coherence length of the radiation source. For a light source 
with a Gaussian spectral distribution, the coherence length is given by:

$l_{\mathrm{coh}}=\frac{2 \ln 2}{\pi} \cdot \frac{\lambda_{0}^{2}}{\Delta \lambda}$,

where $\lambda_{0}$ is the central wavelength and $\Delta \lambda$ is the spectral width of the light source [16]. In the near-infrared regime with a central wavelength of $\lambda_{0}=1 \mu \mathrm{m}$ and a bandwidth of $\Delta \lambda=400 \mathrm{~nm}$, the axial resolution is limited to $1.1 \mu \mathrm{m}$. Although this resolution is sufficient to investigate, for example, retinal structures, imaging of nanoscaled objects like semiconductor structures is out of reach. However, the coherence length and thus the axial resolution of OCT can significantly be improved using radiation with a shorter wavelength.

XUV coherence tomography (XCT) extends optical coherence tomography (OCT) into the extreme ultraviolet (XUV) and soft X-ray (SXR) range, which enables approaching axial resolutions in the nanometer regime [17]. XCT has been demonstrated for the first time with a synchrotron radiation source [18]. Although the applicability of synchrotron-based imaging methods is limited due to troublesome accessibility of the large-scale facilities, it has been shown that three-dimensional imaging of axial nanostructures is feasible [18]. However, in recent years, the rapid development of extremely broad bandwidth laser-driven XUV and SXR sources using the high-harmonic generation (HHG) [19] and laser-plasma sources (LPS) [20, 21] has facilitated the realization of XCT at a laboratory scale [22, 23].

In the XUV and SXR range, the absorption of almost all materials is typically very high. Therefore, cross-sectional imaging is only feasible in spectral transmission windows of the sample's main materials. Luckily, many materials have such transmission windows. Two of them have conspicuous application potential. On one hand, silicon has a transmission window from $\lambda=12 \mathrm{~nm}$ to $40 \mathrm{~nm}$ calling for applications in the semiconductor industry. On the other hand, the well-known water window between $\lambda=2.3 \mathrm{~nm}$ and $4.4 \mathrm{~nm}$ enables penetration of biological samples and offers a unique carbon absorption contrast for imaging [24-26]. The theoretical limit of the axial resolution of XCT in these two ranges is $10 \mathrm{~nm}$ and $3 \mathrm{~nm}$, respectively, according to Eq. 1 .

In this letter, we describe the XCT apparatus and summarize recent results on the non-destructive investigation of nanoscale layered structures using XCT.

\section{Technical implementation}

\subsection{A common-path interferometric apparatus for XCT}

Adapting OCT to operate in the XUV/SXR range requires severe modifications of the Michelson interferometerbased OCT geometry mainly due to the high absorption level for these spectral ranges. A typical two-arm arrangement would require sophisticated broadband optics and, in particular, broadband beam splitters. The fabrication of such beam splitters with nanometer surface flatness is extremely demanding and expensive. Moreover, measuring the interference pattern in the XUV requires nanoscale mechanical stability of the interferometer. Finally, the apparatus must operate under vacuum conditions due to the strong absorption of XUV and SXR radiation in the air.

These technical challenges can be circumvented by employing a common-path reflective interferometric setup. The collinear geometry significantly reduces stability issues and does not require a beam splitter, since the surface of the sample itself serves as a reference. The XCT setup is schematically illustrated in Fig. 1 (left). Broad bandwidth XUV/SXR radiation is typically focused on a sample by a toroidal mirror. Precise positioning of the sample is realized by motorized positioners. The reflected radiation is characterized spectrally by an XUV spectrometer [28-30] in case of a broad bandwidth source (spectrometer-based XCT). Alternatively, when spectrally tunable monochromatic radiation is used, the reflectivity can be measured by a photodiode while sweeping the wavelength (swept-source XCT) [18].

\subsection{Principle of axial imaging}

The basic principle underlying the axial imaging using a common-path Fourier-domain scheme is illustrated in Fig. 1 (right). Let us consider a sample consisting of three layers, namely, a capping layer $\mathrm{L}_{1}$ and two buried layers $\mathrm{L}_{2}$ and $\mathrm{L}_{3}$ located at depths $\mathrm{L}_{12}$ and $\mathrm{L}_{13}$. Such a system can be treated as a Fabry-Pérot interferometer (FPI). The reflection from the capping layer $\left(\mathrm{L}_{\text {ref }}\right)$ defines the reference arm and a reflection from a deeper layer $\left(\mathrm{L}_{\text {probe }}\right)$ and the signal arm and both propagate along the same path [31]. When the sample is irradiated with broad bandwidth XUV radiation, the reflected spectrum exhibits well-defined modulation frequencies $\Delta \omega$ depending on the depths of the layers. According to the Wiener-Khinchin theorem, the Fourier transform of the reflected spectrum equals the autocorrelation of the field reflectance, which encodes the depth structure [32]. The depth location of the layers 

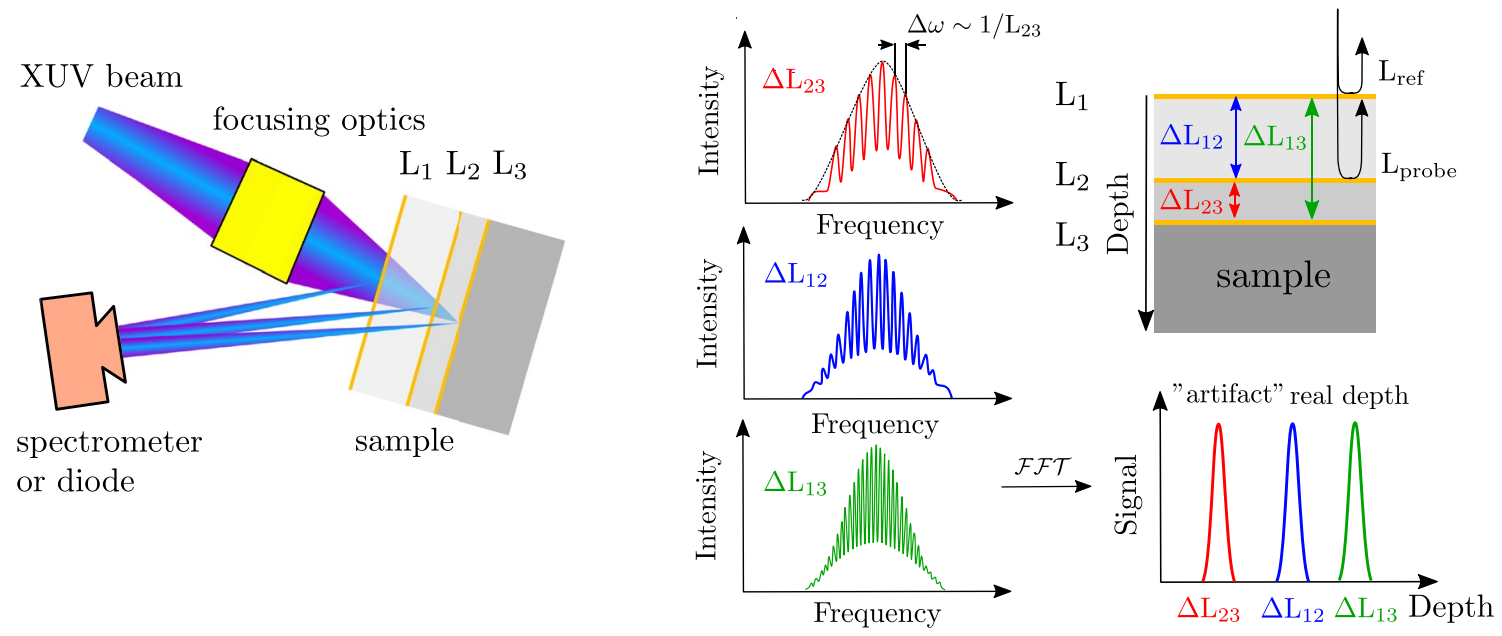

Fig. 1 (Left) A schematic representation of a common-path Fourierdomain XCT interferometer: broad bandwidth XUV/SXR radiation is focused onto the sample mounted on a motorized positioner [27]. The reflected light from the sample is detected by an XUV spectrometer or by a photodiode. (Right) Principle of the depth measurement with common-path Fourier-domain XCT: a sample consisting of three layers $\mathrm{L}_{1-3}$ is irradiated with broad bandwidth radiation

$\Delta \mathrm{L}_{\mathrm{ij}}$ can be retrieved from the position of the peaks in the autocorrelation signal being proportional to $\Delta \omega \propto 1 / \Delta \mathrm{L}_{\mathrm{ij}}$. However, the refractive index of the dominant host material and the incident angle have to be taken into account to precisely reconstruct the position of the particular layers. In the common-path Fourier-domain XCT setup only the reflected spectral intensity can be measured. Therefore, the phase information is lost [22]. Assuming that the field of the radiation source is:

$\bar{E}_{\text {source }}(\omega)=E_{\text {source }} e^{i \varphi_{\text {source }(\omega)}}$,

the reflected field is defined as:

$\bar{E}_{\mathrm{refl}}(\omega)=E_{\mathrm{refl}} e^{i \varphi_{\mathrm{refl}}(\omega)}=\bar{E}_{\mathrm{source}}(\omega) \cdot \bar{r}(\omega)$.

Here, $E(\omega)$ is the electric field in the frequency domain, $\varphi$ is the phase, and $r(\omega)$ is the field reflectivity. In the experiment, the spectrometer measures the spectrum given by:

$S_{\mathrm{M}}(\omega)=\left|\bar{E}_{\mathrm{refl}}(\omega)\right|^{2}$.

The intensity reflectivity of the sample can be calculated if the spectrum of the light source $S_{\text {source }}(\omega)$ is determined by measuring the reflected spectrum of a known sample (e.g., a blank silicon wafer):

$R(\omega)=|\bar{r}(\omega)|^{2}=S_{\mathrm{M}}(\omega) / S_{\text {source }}(\omega)$.

The Fourier transform of the intensity reflectivity $R(\omega)$ leads to the autocorrelation of the structure sample. It not only contains the position of deeper layers with respect to the
(Gaussian spectral envelope). The sample can be regarded as a two nested Fabry-Pérot-type interferometers. An interference between the surface $\left(\mathrm{L}_{\mathrm{ref}}\right)$ and the buried layers $\left(\mathrm{L}_{\text {probe }}\right)$ modulates the spectrum with a frequency being proportional to the distance between layers, e.g., $\Delta \omega \sim 1 / \Delta \mathrm{L}_{23}$. The depth structure can be obtained by a Fourier transform (FT) of the measured reflected spectrum

top layer $\left(\Delta \mathrm{L}_{12}, \Delta \mathrm{L}_{13}\right)$ but also with respect to all other layers-in this case, $\mathrm{L}_{2}\left(\Delta \mathrm{L}_{23}\right)$. The latter one is referred to as an artifact or "ghost peak". However, a recently developed iterative three-step phase-retrieval algorithm (PR-XCT) [22] based on a Gerchberg-Saxton [33] and a hybrid input-output approach [34] can be used to computationally reconstruct the unknown phase. Thus, the algorithm is capable of an unambiguous reconstruction of the sample's axial structure by eliminating the artifacts very efficiently.

\subsection{Radiation sources}

So far, XCT has been demonstrated with three different types of XUV/SXR sources. The first proof-of-concept experiment was performed with synchrotron radiation [18]. Later, XCT has as well be realized using laser-based high-harmonic generation (HHG) [22] and laser-plasma sources (LPS) [23].

The experiments employing synchrotron radiation have been performed at the BW3 wiggler beamline of the DORIS III storage ring at DESY in Hamburg (silicon window) [35] and the UE-112-PGM1 undulator beamline at the BESSY II storage ring in Berlin (water window) [36]. A broad spectrum of XUV radiation in the silicon transmission window $(\Delta E=30-100 \mathrm{eV})(40-112 \mathrm{~nm})$ enabling spectrometer-based XCT was provided using the monochromator of the beamline in 0th diffraction order. In the water window $(\Delta E=280-530 \mathrm{eV})(4.30-2.3 \mathrm{~nm})$, in contrast, sweptsource XCT was realized by scanning the wavelength in steps of $1 \mathrm{eV}$ with the beam line's monochromator. 
Using the high-harmonic generation (HHG) [37, 38], XCT was be realized on the laboratory scale for the first time. To this end, a quasi-supercontinuous HHG source has been implemented. Since, in XCT the depth, information of the sample is encoded in spectral modulations, the intrinsic modulation of the HHG spectrum needs to be suppressed. This could be accomplished by rapidly sweeping the wavelength of the driving laser. As a consequence, the harmonic comb is shifted as well and, on time-average, a supercontinuum is generated. Technically, this has been realized with a commercial three-stage optical parametric amplifier (OPA) system. The signal output beam is focused with a lens $(f=30 \mathrm{~cm})$ down to intensities of $10^{14} \mathrm{~W} / \mathrm{cm}^{2}$ into an argon-filled nickel tube at backing pressures around 300 mbar. The phase-matching conditions are optimized by controlling the gas pressure and lens position. The XUV supercontinuum is generated by sweeping the driving OPA wavelength in the range of $1.22-1.32 \mu \mathrm{m}$ (in $10 \mathrm{~nm}$ steps). Such small variations of the wavelength are sufficient to shift the harmonic comb and fill the gaps in the HHG spectrum [19]. To filter out the XUV radiation from the remaining laser light, a $200 \mathrm{~nm}$ aluminum foil transmitting in the range of $33-72 \mathrm{eV}$ has been used. This slightly reduced the accessible bandwidth in the silicon window and in consequence the axial resolution.

Laboratory-based XCT in the water window has been realized very recently using laser-plasma radiation. In the experiment, $3 \mathrm{~ns}$ long pulses from a Nd:YAG laser system (NL $303 \mathrm{HT}$, EKSPLA) with a pulse energy of $0.65 \mathrm{~J}$ are focused with a lens $(f=2.5 \mathrm{~cm})$ into a dense gas jet from a double-stream gas puff target $[39,40]$. In the gas, a laser plasma is created and emits radiation ranging from XUV down to the SXR range. The target consists of an inner and a surrounding outer gas. The inner gas serves as the actual source of the radiation to which a specific elemental emission can be attributed. The outer gas limits the expansion of the target gas. Consequently, the target density in the interaction region is significantly increased and the XUV/ SXR emission is enhanced. To generate SXR radiation, the inner nozzle has been supplied with krypton at a pressure of $p=4$ bar. The krypton plasma efficiently emits radiation in the spectral range between $\lambda=2.2 \mathrm{~nm}$ and $5.5 \mathrm{~nm}$ covering the entire water window. The outer nozzle was supplied with helium at a pressure of $p=5.5 \mathrm{bar}$.

\section{Experimental results}

\subsection{XCT in silicon window with synchrotron radiation and HHG}

Two samples were designed to deliver the proof-of-concept XCT experiment in the silicon transmission window, as illustrated in Fig. 2a and d. The sample in Fig. 2a consists of one thin layer of gold with $5 \mathrm{~nm}$ thickness, which is buried under a $170 \mathrm{~nm}$ layer of amorphous silicon. The sample in Fig. 2d contains two $5 \mathrm{~nm}$-thick gold layers spaced by $30 \mathrm{~nm}$ and buried under $100 \mathrm{~nm}$ of silicon. The samples were prepared by sputtering gold and silicon layers onto a silicon substrate. A capping layer on the top of the sample was formed by a natively grown few nanometer thick $\mathrm{SiO}_{2}$ layer upon contact of silicon with the ambient atmosphere. The position of the samples is controlled by a piezo-driven motorized stage during the measurement. Synchrotron-based as well as HHG-based XCT measurements have been performed on both samples and allow a comparison.

The reflected spectral intensity recorded for both samples for both sources is shown in Fig. 2b and d, respectively. The energy scale is converted into $\kappa_{D}$-axis by including the dispersion of the dominating material $n_{D}(E)$ (in this case silicon) and the angle of incidence $\alpha$ using relation:

$\kappa_{D}=\frac{2 E}{\hbar c} \sqrt{n_{D}^{2}(E)-\sin ^{2} \alpha}$.

The synchrotron measurement was performed for a wider energy range $(32-99 \mathrm{eV}$, blue) than the measurement employing HHG radiation where the bandwidth is limited by the transmission of the aluminum filter $(37-72 \mathrm{eV}$, red). To avoid ringing artifacts in the Fourier transform, the spectra are weighted by a Kaiser-Bessel window (dashed lines). The small amplitude deviations in the reflectivity are due to the spectrometer adjustment and degradation of the samples in the time between the measurements. The laser intensity jitter introduced noise due to the high nonlinearity of the HHG process, which could be resolved in the measurement with the two-layer system.

The right panel of Fig. 2 shows the XCT signal (dot-dashed lines) resulting from the Fourier transform of the spectral reflectivity ( $c$ and $\mathrm{f}$ ). The positions of the peaks in the XCT signal representing the depth positions of the gold layers $(170 \mathrm{~nm}$ for the single layer, and 110 and $140 \mathrm{~nm}$ for the double layer) are in very good agreement in both data sets. The main limitation for precise determination of the position of the layers $( \pm 4 \mathrm{~nm})$ is the measurement error of the angle of incidence $\left( \pm 1^{\circ}\right)$ and remaining dispersion effects of the gold layers. The peak around $140 \mathrm{~nm}$ in the laser-based XCT (red, Fig. 2 f) is slightly shifted when compared to the position retrieved by the synchrotron measurement (blue). This can be explained by sample degradation processes, since two measurements were taken with several months in between. It is known that thin gold layers tend to diffuse into silicon, thus altering the sample structure over long periods of time. The 


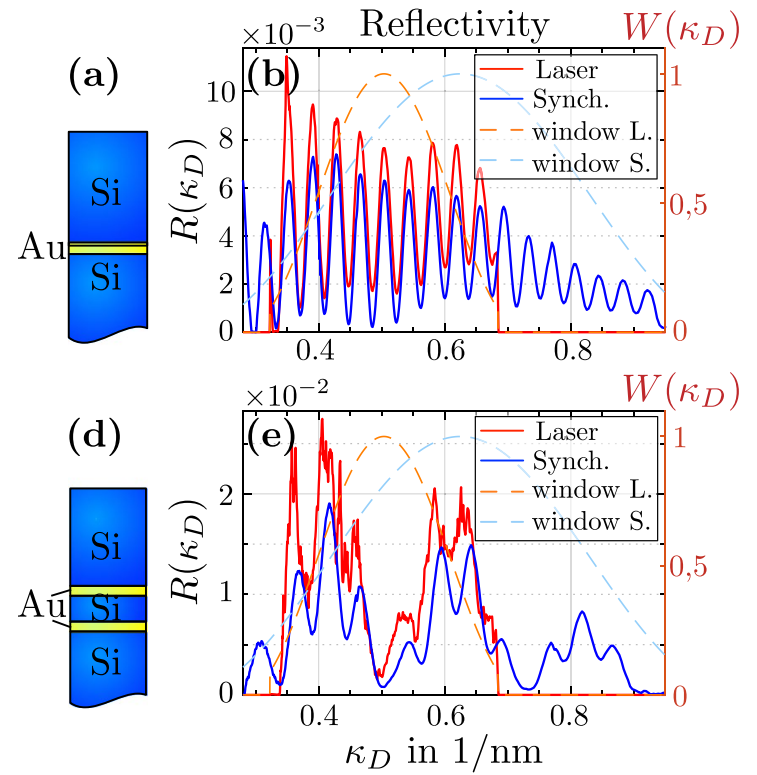

Fig. 2 Results of XCT measurements in the silicon transmission window: the samples consist of a single (a) and double (d) thin layers of gold with $5 \mathrm{~nm}$ thickness, which are buried under $170 \mathrm{~nm}$ and $100 \mathrm{~nm}$ layer of amorphous silicon, respectively. The measured reflectivities $\left(R\left(\kappa_{D}\right)\right)$ for each sample in the silicon transmission window are obtained with synchrotron radiation $(32-99 \mathrm{eV}$, blue $)$ and HHG source $(37-72 \mathrm{eV}$, red). Small amplitude deviations are due to the spectrometer adjustment and degradation of the samples. The energy scale is converted into $k_{D}$-axis by including dispersion of the dominating material (silicon) and the angle of incidence. The dashed curves represent the Kaiser-Bessel spectral windows used to suppress the Fourier artifacts. The right panel (c and f) shows the reconstructed depth profile (dot-dashed lines) by the Fourier transform of experimentally measured reflectivity. The positions of peaks $(170 \mathrm{~nm}$ for

axial resolution is determined based on the Rayleigh criterion and the Fourier transform of the filter window. In all cases, the nanometer range is clearly reached as expected for XCT. For the HHG experiment, the axial resolution is about $29 \mathrm{~nm}$, which is slightly lower than in the experiment with synchrotron radiation $(15 \mathrm{~nm})$ due to the reduced bandwidth of the HHG source.

Since in the original XCT signal the lack of phase information leads to autocorrelation artifacts and thus to an ambiguous signal, one can identify two ghosts in the signal. First, the strong ghost peak which appears in the measurement of the double-layer sample at a depth of $30 \mathrm{~nm}$ corresponds to the depth difference between the two gold layers. Second, the peak at zero depth is the sum of a reflection from the sample's surface and the DC component of the radiation reflected from buried layers. With a novel phase-retrieval algorithm (PR-XCT), the ambiguities can be eliminated efficiently [22]. As depicted in Fig. 2c and $\mathrm{f}$, the ghost peak and the DC components are almost completely suppressed. As a result, the real sample structure including the surface reflectivity could be reconstructed
PR-XCT signal

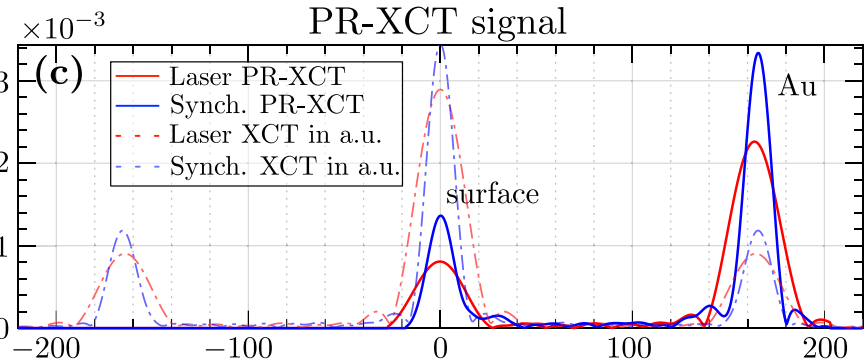

$\times 10^{-3}$

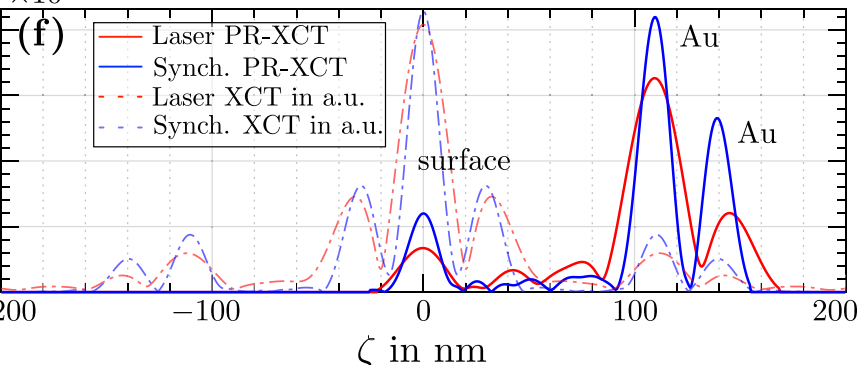

the single layer, $110 \mathrm{~nm}$ and $140 \mathrm{~nm}$ for the double layer) determine the depths of the buried gold layers and are in very good agreement in both data sets. The "ghost peak" at $30 \mathrm{~nm}$ (f) is an autocorrelation artifact, commonly referred to as "autocorrelation" in OCT imagery. Its position corresponds to the difference in depths between two gold layers in the sample (d). Note that the amplitude of peak at $\zeta=0 \mathrm{~nm}$ consists of the reflectivity of surface and DC components resulting from the Fourier transform. The solid lines display the results of the retrieved phase data using the PR-XCT algorithm which eliminates the autocorrelation artifacts. Thus, it enables to reconstruct the real reflectivity of the layers, which is encoded in the amplitude of corresponding peaks. The axial resolution determined by the Rayleigh criterion and the Fourier transform of the filter window for synchrotron data is $15 \mathrm{~nm}$ and for $\mathrm{HHG}$ radiation $29 \mathrm{~nm}$

from both data sets independently (solid lines). It should be noted that even the high noise level present in the HHG measurement for the double-layer sample (Fig. 2e) does not affect the reconstruction, because the information is encoded in the modulation frequency. This clearly demonstrates that Fourier-domain XCT is robust against noise caused, e.g., by the laser intensity fluctuations [Fig. 2e (red line)]. Such rapid fluctuations typically occurring on a single laser shot basis generate high-frequency component in the Fourier spectrum. In fact, they filter themselves out from the lower frequency signal modulations carrying information about the sample structure.

\subsection{XCT in the water window with synchrotron radiation}

To demonstrate XCT in the SXR range, an experiment in the water window using synchrotron radiation has been performed. A layered sample consisting of a $8 \mathrm{~nm}$ platinum layer buried under a boron carbide layer has been produced and investigated by XCT. It is depicted in Fig. 3a. 


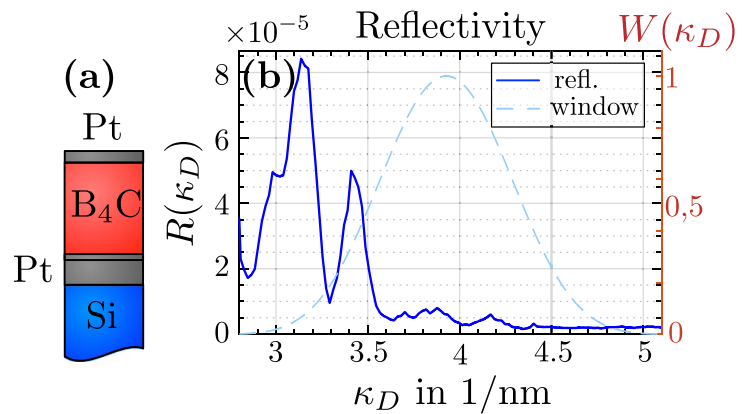

Fig. 3 Results of an XCT investigation in the water transmission window: sample (a) consists of a $8 \mathrm{~nm}$-thick Pt layer buried under $18 \mathrm{~nm}$ of boron carbide $\mathrm{B}_{4} \mathrm{C}$. b Recorded reflected spectral intensity in the water window $280-530 \mathrm{eV}(4.4-2.3 \mathrm{~nm})$ of the layer system. The solid line (blue) represents the interpolation of data acquired for different monochromator positions. The dashed curve (light blue) is the Kaiser-Bessel spectral window used to suppress Fourier artifacts. c Reconstructed depth profile (dot-dashed line) by Fourier transform of experimentally measured reflectivity. The frontside and backside of the platinum layer appear separated, and thus, the resolution is better than $8 \mathrm{~nm}$. The first peak at $7.7 \mathrm{~nm}$ can be identified as an artifact, which corresponds to the difference between the two real depths. The blue solid line represents the results of the phase retrieval with PRXCT algorithm. The PR-XCT suppresses the autocorrelation artifact at $7.7 \mathrm{~nm}$ and retrieves the layer's reflectivity, which is encoded in the peak amplitudes. The axial resolution determined by the Rayleigh criterion and the Fourier transform of the filter window is $7 \mathrm{~nm}$
The measured reflectivity captured by swept-source XCT is shown in Fig. 3b. The blue line represents the interpolation of measurements acquired for different monochromator positions. In analogy to the previous data in the silicon window, the spectrum is multiplied by a filter function (dashed line). The XCT signal (c, dot-dashed curve) shows three distinct peaks. The peaks at $17 \mathrm{~nm}$ and $25 \mathrm{~nm}$ represent the position of the frontside and backside of the platinum layer, respectively. The peak at $7.7 \mathrm{~nm}$ is the autocorrelation artifact representing the difference in depth of the frontside and backside of the platinum layer. With the PR-XCT, it is possible to eliminate the ghost artifacts and reconstruct the real sample reflectivity. Note that in contrast to the siliconwindow measurement, the amplitude of the peak at $\zeta=0 \mathrm{~nm}$ is much higher due to the higher reflectivity of the platinum capping layer. The axial resolution is defined by the bandwidth and the filter function and is as high as $7 \mathrm{~nm}$ (FWHM). The two layers with a distance of $8 \mathrm{~nm}$ are clearly separated demonstrating XCT can achieve single-digit nanometer axial resolution.

\subsection{XCT in the water window with laser-plasma radiation source (LPS)}

To perform laboratory-based XCT in the SXR range, a broad bandwidth SXR continuum generated by the laserplasma source (LPS) has been used [23]. In this experiment, a standard Mo/Si mirror designed for unpolarized $13.5 \mathrm{~nm}$ VUV radiation with a spectral bandwidth of $1 \mathrm{~nm}$ and a reflectivity of $\sim 37 \%$ (Fraunhofer IOF) was used as a sample. It is composed of 40 pairs of Si/Mo multilayers deposited on top of a $\mathrm{SiO}_{2}$ substrate. According to the technical specifications of the mirrors, the thickness of the layers is $6 \mathrm{~nm}$ for silicon and $4 \mathrm{~nm}$ for molybdenum, as illustrated in Fig. 4a. The sample is illuminated by the broadband SXR radiation $(\lambda=2-5 \mathrm{~nm})$ from the LPS $\left(\mathrm{I}_{\text {inc }}(\lambda)\right)$. The sample reflects the SXR radiation at $45^{\circ}$ towards a grazing-incidence SXR spectrometer $\left(\mathrm{I}_{\text {ref }}(\lambda)\right)$. Further details on the experimental apparatus can be found in [23].

The measured spectral reflectivity of the sample is converted into $\kappa_{D}$-space (teal curve in Fig. $4 \mathrm{~b}$ ) and windowed with a Gaussian function (light green curve, parameters are $\kappa_{0}=2.6 \mathrm{~nm}^{-1}$ and FWHM width $\sigma=1.8 \mathrm{~nm}^{-1}$ ) as shown in Fig. 4 (b, orange curve). The corresponding XCT signal, i.e., the Fourier transform, is shown in Fig. $4 \mathrm{c}$ and reveals depth information from the Mo/Si structure (bottom, pink curve). It is compared to a simulation (top, blue curve) of the XCT signal calculated via the matrix method based on Fresnel's equations. The numbers, above and below the curves, indicate the horizontal position (depth values) of each peak for comparison between theoretical and experimental data. Both are in good agreement. The main peaks, spaced every period of the multilayer structure, are clearly matched. The positions of the measured peaks in between the main peaks, for depths larger than $10 \mathrm{~nm}$, correspond well to the simulated data; however, a shift of $\sim 1-2 \mathrm{~nm}$ is present. Considering the spatial frequency limit of the Gaussian window function, used in Fig. 4b in terms of its Fourier transform, the FWHM width of the Gaussian window is $2.2 \mathrm{~nm}$. This corresponds to the FWHM width of the narrowest peak in the reconstructed axial profile, as shown in Fig. 4c. Due to high periodicity of the sample, a finite depth constraint for the phase-retrieval (PR-XCT) is not possible and thus the algorithm does not converge stably. This problem has to be addressed in the future. 

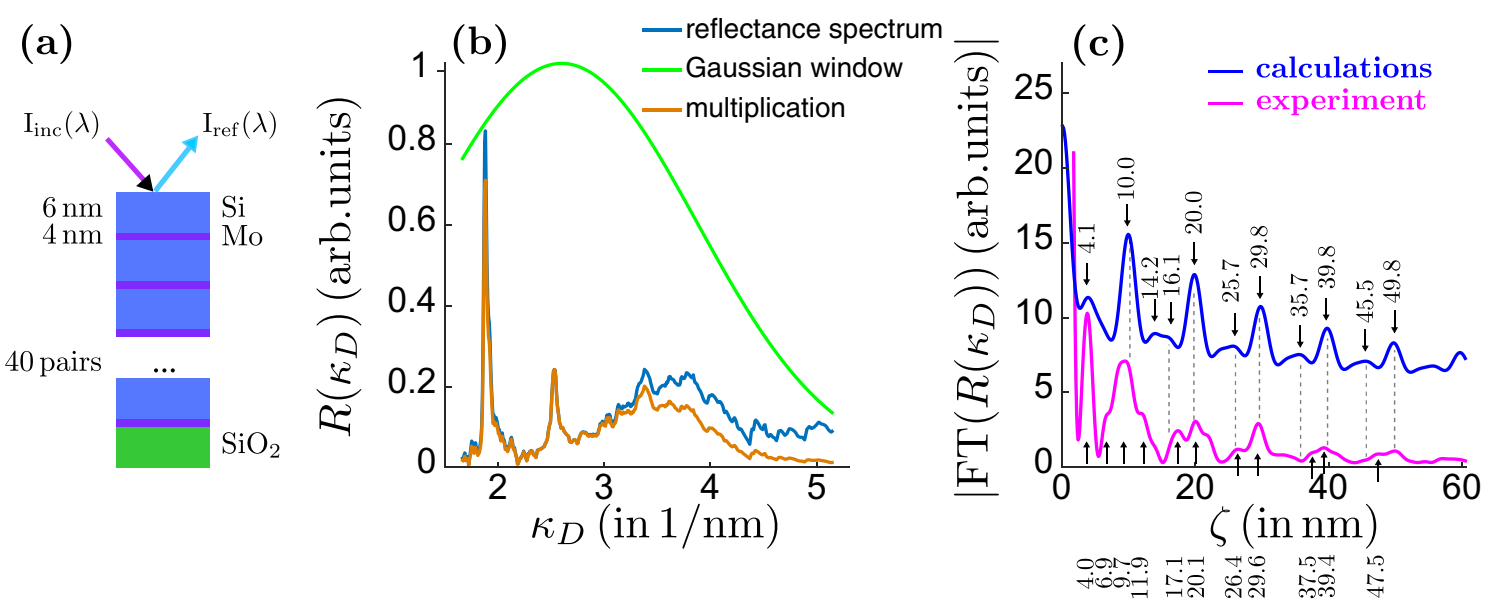

Fig. 4 Schematic of the $\mathrm{Mo} / \mathrm{Si}$ multilayer structure studied using "water-window" XCT (a). The sample consists of 40 pairs of $6 \mathrm{~nm}-$ thick silicon and $4 \mathrm{~nm}$-thick molybdenum layers deposited on a $\mathrm{SiO}_{2}$ substrate. b The measured reflectivity (teal line) is filtered with a Gaussian-type window function (green) to suppress the Fourier arti- facts. The orange line represents the multiplication of both curves. A reconstruction of the depth information from the $\mathrm{Mo} / \mathrm{Si}$ structure, experimental data (bottom, pink curve), and simulation (top, blue curve) (c), with numbers indicating the peak positions
Table 1 Comparison of different technological approaches presented in this review. Features are grouped according to the radiation source used in the experiment, i.e., table-top laser systems (HHG and LPS) and the synchrotron source. Look the discussion for further details

\begin{tabular}{lllll}
\hline $\begin{array}{l}\text { XCT } \\
\text { approach }\end{array}$ & $\Delta \lambda(\mathrm{nm})$ & $\begin{array}{l}\text { Axial }^{(3)} \\
\text { resolution }\end{array}$ & Photon flux $(\mathrm{ph} / \mathrm{s})$ & Comments \\
\hline Table-top system & $33.5-17.22^{(1)}$ & $29^{(4)}$ & $4 \cdot 10^{9(6)}$ & $\begin{array}{l}\text { Broad-bandwidth spectrum, } \\
\text { accessibility, time resolution }\end{array}$ \\
Synchrotron & $5-2^{(2)}$ & $2.2^{(4)}$ & $10^{10^{(7)}}$ & $10^{12^{(8)}}$ \\
& $38.75-12.52$ & $15^{(4)}$ & $\begin{array}{l}\text { High flux } \\
\text { widely tunable energy range }\end{array}$ \\
\hline
\end{tabular}

HHG source, (2) LPS source, (3) in nm; limited by width of the spectral filter, (4) Kaiser-Bessel window, (5) Gaussian window, (6) [27], (7) [41, 42], and (8) e.g., $\Delta \mathrm{E}=20-280 \mathrm{eV}$ [36], $\Delta \mathrm{E}=250-3000 \mathrm{eV}$ [43],

\section{Discussion}

In Table 1, we summarize the most important features of different realizations of the coherence tomography experiments in the XUV/SXR regime. The features are grouped according to the type of the radiation source used in the experiments. The table-top laser-based sources based on the HHG process (XCT in Si window) and LPS (XCT in the water window) are compared with results obtained using the synchrotron radiation (PETRA III). The following discussion concentrates on the most essential features of each approach.

This review has concentrated on the axial imaging of the layered samples. According to Eq. 1, the axial resolution in the coherence tomography experiment depends solely on the central wavelength $(\lambda)$ and the square of the bandwidth of the light source $\left(\Delta \lambda^{2}\right)$. However, to avoid ringing artifacts in the Fourier transform, the spectra are weighted by the spectral window, e.g., Kaiser-Bessel window (Figs. 2a, d, 3a) and Gaussian window (Fig. 4b). We emphasize that in all presented results, the achieved axial resolution is limited by its width. Improving of the axial resolution can be achieved by controlling the bandwidth and, thus, the width of the filtering function. This can be realized in several ways taking into account the sample material transmission windows. In the HHG process, the high-energy cut-off scales as $3.17 \mathrm{Up}$ with the ponderomotive energy defined as Up $\sim \mathrm{I} \cdot \lambda^{2}$ and can be extended by increasing the laser intensity or preferably the wavelength of the optical field. Technically, the applicable bandwidth is further limited by the transmission of the filters used to attenuate optical field driving the HHG process. The shaping of the spectrum of the LPS source, in turn, can straightforwardly be realized by mixing various gases. However, it is limited by the atomic structure of the target gas. The experiments with the synchrotron radiation offer the most versatile controlling of the wavelength. By its 
nature, the synchrotron radiation typically delivers monochromatic radiation ranging from VUV to hard X-ray at high flux $\left(10^{11} \mathrm{ph} / \mathrm{s}\right)$.

When it comes to available photon flux and thus fast image acquisition, synchrotrons are still the most powerful sources. For example the PETRA III synchrotron facility (station P04) delivers up to $10^{12}$ photons $/ \mathrm{s} / 0.1 \% \mathrm{BW}$ in the entire accessible range [43]. However, their limited accessibility and the large costs severely restrict the applicability of synchrotron-based XUV/SXR imaging. A standard table-top HHG laser source delivers $10^{9} \mathrm{ph} / \mathrm{s}$ [27] in the XUV range. Although extending the cutoff of the HHG spectrum towards SXR range can be realized using mid-infrared wavelength, it happens at unavoidable costs of the photon flux. The conversion efficiency of the HHG process is limited by phasematching conditions and reaches $10^{-3}-10^{-4}$ in the VUV range and $10^{-6}-10^{-7}$ in the SXR range [44]. This problem can be circumvented by the development of high average power laser systems $[45,46]$, which are suited for various types of the imaging. In the mean time, LPS sources based on a double-stream gas-puff system are an attractive alternative to generate SXR continuum. They require relatively low-cost and very stable nanosecond drivers. These sources deliver enough photons $\left(10^{10} \mathrm{ph} / \mathrm{s}\right)$ in the water window to enable laboratory-sized SXR imaging [41, 42]. Further increasing of the photon flux of LPS sources can be realized using high-repetition rate nanosecond drivers.

However, like in OCT, lateral imaging can be added to XCT by repeating the depth measurement with a focused illumination in a scanning approach. The lateral resolution is therefore limited by the NA of the focusing optics, but the axial resolution remains only dependent on the coherence length of the light source and is thus independent on the focusing. This is a considerable advantage especially in the XUV range, where high-NA focusing is extremely demanding and expensive. Although not shown in this article, the XCT implementations at synchrotrons and HHG sources already included lateral imaging by using toroidal mirrors. These reflective grazing-incidence optics provide a lateral resolution of a few tens of micrometers. Further improvement of the lateral resolution of XCT requires better focusing optics with more advanced components such as multiple toroidal mirrors [47], Kirkpatrick-Baez Optics [48], or zone plates [49]. Alternatively, a mask with a small hole in front of the sample can be used to limit the area of interaction and thus increasing the lateral resolution at the expense of the usable photon flux.

Although, in the water window, the synchrotrons are still much more powerful, laser-based approaches begin catching up $[44,50]$. They have been employed for various nanometer imaging techniques such as SXR microscopy, X-ray diffraction imaging, and X-ray holography, which utilize rather narrow bandwidth XUV/SXR radiation. The XCT makes efficient use of the intrinsically broad spectrum of laser-based HHG and LPS sources. This is in contrast to the experiments using the synchrotron radiation that require the wavelength sweeping over the broad energy ranges. Besides the superior accessibility of laser-based sources, their intrinsic time resolution is another advantage. In the case of $\mathrm{HHG}$, it can even reach the attosecond range allowing the investigation of ultrafast processes.

In comparison to a freestanding interferometric setup with a distinct reference arm, the common-path XCT geometry significantly reduces the technical complexity of the experiment. The axial structure of the sample can be reconstructed solely by a Fourier transform of the reflected spectrum from the sample (common-path Fourier-domain XCT). However, this simplification comes at unavoidable costs. Without the distinct reference arm, the spectral phase of the reflected light is lost. As a result, the autocorrelation artifacts appear in the XCT signal and lead to ambiguities in the reconstructed depth information. Computationally, the problem of the autocorrelation artifacts can be approached by adapting a solution from digital optics. Using one-dimensional iterative phase-retrieval algorithms, we were able to reconstruct the phase. However, in comparison to the twodimensional phase-retrieval problem, the one-dimensional one is a by far less over-determined. One needs to make sure that an algorithm is able to find the right physical solution out of many possible ones. By implementing a novel threestep phase-retrieval algorithm, it has been shown that the lost phase of XCT can indeed be reconstructed, which in consequence efficiently removes the autocorrelation artifacts commonly referred to as "autocorrelation" in OCT imagery. The phase-retrieval algorithm (PR-XCT) [22] successfully retrieves the phase of the scattered light from samples containing single and double buried layers (Fig. 2, 3). However retrieving the phase of the light reflected from the multilayered and periodic sample, as shown in Fig.4, requires further development of the numerical algorithm.

Even in its current implementation, where lateral resolution is limited to the micrometer scale, XCT has many possible applications, for example, the investigation of (multilayer-) coatings of optical mirrors or even XUV-mirrors, axial-structured devices like solar cells, or axial-structured semiconductor devices. In addition, imaging of laterally homogeneous biological membranes might be possible. Future XCT generations with high numerical aperture, and thus, high lateral resolution could even have further applications, e.g., non-destructive three-dimensional imaging of three-dimensional semiconductor devices, lithographic masks, and complex biological structures.

Acknowledgements This work was partially supported by the Deutsche Forschungsgemeinschaft (DFG) (projects: PA 730/5 and PA 730/9-1), the Bundesministerium für Bildung und Forschung 
(BMBF) (project VIP "X-Coherent T" and FSP 301-FLASH), the Thüringer Aufbaubank (2015FGR0094), the National Science Centre (NCN) (projects: 2015/19/B/ST3 /00435, 2016/23/G/ST2/04319), and European Union's Horizon 2020 research and innovation program Laserlab-Europe IV (654148).

Funding Open Access funding enabled and organized by Projekt DEAL.

Open Access This article is licensed under a Creative Commons Attribution 4.0 International License, which permits use, sharing, adaptation, distribution and reproduction in any medium or format, as long as you give appropriate credit to the original author(s) and the source, provide a link to the Creative Commons licence, and indicate if changes were made. The images or other third party material in this article are included in the article's Creative Commons licence, unless indicated otherwise in a credit line to the material. If material is not included in the article's Creative Commons licence and your intended use is not permitted by statutory regulation or exceeds the permitted use, you will need to obtain permission directly from the copyright holder. To view a copy of this licence, visit http://creativecommons.org/licenses/by/4.0/.

\section{References}

1. D. Huang, E.A. Swanson, C.P. Lin, J.S. Schuman, W.G. Stinson, W. Chang et al., Optical coherence tomography. Science 254(5035), 1178-1181 (1991)

2. W. Drexler, J.G. Fujimoto, Optical Coherence Tomography (Springer International Publishing, Switzerland, 2015)

3. D. Huang, J.P. Wang, C.P. Lin, C.A. Puliafito, J.G. Fujimoto, Micron-resolution ranging of cornea anterior-chamber by optical reflectometry. Lasers Surg. Med. 11(5), 419-425 (1991)

4. K. Takada, I. Yokohama, K. Chida, J. Noda, New measurement system for fault location in optical waveguide devices based on an interferometric technique. Appl. Opt. 26, 1603-1606 (1987)

5. P.A. Keane, P.J. Patel, S. Liakopoulos, F.M. Heussen, S.R. Sadda, A. Tufail, Evaluation of age-related macular degeneration with optical coherence tomography. Surv. Opthalmol. 57(5), 389-414 (2012)

6. M. Wojtkowski, B.L. Sikorski, I. Gorczyńska, M. Gora, M. Szkulmowski, D. Bukowska et al., Comparison of reflectivity maps and outer retinal topography in retinal disease by 3 -D Fourier domain optical coherence tomography. Opt. Expr. 17(5), 4189-4207 (2009)

7. W.J. Walecki, K. Lai, V. Souchkov, P. Van, S.H. Lau, A. Koo, Novel noncontact thickness metrology for backend manufacturing of wide bandgap light emitting devices. Phys. Status Solidi C 2(3), 984-989 (2005)

8. J. Czajkowski, T. Fabritius, J. Ulański, T. Marszałek, M. GazickiLipman, A. Nosal et al., Ultra-high resolution optical coherence tomography for encapsulation quality inspection. Appl. Phys. B. 21(105), 649 (2011)

9. L. Thrane, T.M. Jørgensen, M. Jørrgensen, F.C. Krebs, Application of optical coherence tomography (OCT) as a 3-dimensional imaging technique for roll-to-roll coated polymer solar cells. Sol. Energ. Mat. Sol. Cells. 97, 181-185 (2012)

10. P. Targowski, B. Rouba, M. Wojtkowski, A. Kowalczyk, The application of optical coherence tomography to non-destructive examination of museum objects. Stud. Conserv. 49(2), 107-114 (2004)

11. H. Liang, M.G. Cid, R.G. Cucu, G.M. Dobre, A.G. Podoleanu, J. Pedro et al., En-face optical coherence tomography—a novel application of non-invasive imaging to art conservation. Opt. Express 13(16), 6133-6144 (2005)

12. T. Arecchi, M. Bellini, C. Corsi, R. Fontana, M. Materazzi, L. Pezzati. et al. Optical coherence tomography for painting diagnostics. In: Salimbeni R, Pezzati L, editors. Optical Methods for Arts and Archaeology. vol. 5857. International Society for Optics and Photonics. SPIE. pp. 278 - 282. (2005)

13. P. Targowski, M. Iwanicka, Optical Coherence Tomography: its role in the non-invasive structural examination and conservation of cultural heritage objects-a review. Appl. Phys. A 106(2), 265277 (2012)

14. M.L. Yang, C.W. Lu, I.J. Hsu, C.C. Yang, The use of optical coherence tomography for monitoring the subsurface morphologies of archaic jades. Archaeometry 46, 171-182 (2004)

15. R.C. Youngquist, S. Carr, D.E.N. Davies, Optical coherencedomain reflectometry: a new optical evaluation technique. Opt. Lett. 12(3), 158-160 (1987)

16. J.G. Fujimoto, C. Pitris, S.A. Boppart, M.E. Brezinski, Optical Coherence Tomography: an emerging technology for biomedical imaging and optical biopsy. Neoplasia. 2(1), 9-25 (2000)

17. S. Fuchs, A. Blinne, C. Rödel, U. Zastrau, V. Hilbert, M. Wünsche et al., Optical coherence tomography using broad-bandwidth XUV and soft X-ray radiation. Appl. Phys. B. 106, 789 (2012)

18. S. Fuchs, C. Rödel, A. Blinne, U. Zastrau, M. Wünsche, V. Hilbert et al., Nanometer resolution optical coherence tomography using broad bandwidth XUV and soft x-ray radiation. Sci. Rep. 6, 20658 (2016)

19. M. Wünsche, S. Fuchs, S. Aull, J. Nathanael, M. Möller, C. Rödel et al., Quasi-supercontinuum source in the extreme ultraviolet using multiple frequency combs from high-harmonic generation. Opt. Express 25(6), 6936-6944 (2017)

20. H. Fiedorowicz, A. Bartnik, R. Jarocki, R. Rakowski, M. Szczurek, Enhanced X-ray emission in the 1-keV range from a laserirradiated gas puff target produced using the double-nozzle setup. Appl. Phys. B. 70(2), 305-308 (2000)

21. P. Wachulak, A. Bartnik, H. Fiedorowicz. Nanoimaging using a compact laser plasma soft $\mathrm{x}$-ray source based on a gas puff target. In: Lai B, Somogyi A, editors. X-Ray Nanoimaging: Instruments and Methods IV. vol. 11112. International Society for Optics and Photonics. SPIE p. 117. (2019)

22. S. Fuchs, M. Wünsche, J. Nathanael, J.J. Abel, C. Rödel, J. Biedermann et al., Optical coherence tomography with nanoscale axial resolution using a laser-driven high-harmonic source. Optica. 4(8), 903-906 (2017)

23. P. Wachulak, A. Bartnik, H. Fiedorowicz, Optical coherence tomography (OCT) with $2 \mathrm{~nm}$ axial resolution using a compact laser plasma soft X-ray source. Sci. Rep. 8, 8494 (2018)

24. J. Kirz, D. Sayre. In: Winick H, Doniach S, editors. Soft X-Ray Microscopy of Biological Specimens. Boston, MA: Springer US p. 277-322 (1980)

25. J. Kirz, C. Jacobsen, M. Howells, Soft X-ray microscopes and their biological applications. Q. Rev. Biophys. 28(1), 33-130 (1995)

26. Berglund, Rymell, Peuker, Wilhein, Hertz, Compact water-window transmission X-ray microscopy. Compact water-window transmission X-ray microscopy 197, 268-273 (2000)

27. J. Nathanael, M. Wünsche, S. Fuchs, T. Weber, J.J. Abel, J. Reinhard et al., Laboratory setup for extreme ultraviolet coherence tomography driven by a high-harmonic source. Rev. Sci. Instrum. 90(11), 113702 (2019). https://doi.org/10.1063/1.5102129

28. J. Jasny, U. Teubner, W. Theobald, C. Wulker, J. Bergmann, F.P. Schfer, A single-shot spectrograph for the soft-X-ray region. Rev. Sci. Instr. 65(5), 1631-1635 (1994)

29. S. Fuchs, C. Rödel, M. Krebs, S. Hädrich, J. Bierbach, A.E. Paz et al., Sensitivity calibration of an imaging extreme ultraviolet spectrometer-detector system for determining the efficiency of 
broadband extreme ultraviolet sources. Rev. Sci. Instr. 84, 023101 (2013)

30. M. Wünsche, S. Fuchs, T. Weber, J. Nathanael, J.J. Abel, J. Reinhard et al., A high resolution extreme ultraviolet spectrometer system optimized for harmonic spectroscopy and XUV beam analysis. Rev. Sci. Instr. 90(2), 023108 (2019)

31. A.B. Vakhtin, D.J. Kane, W.R. Wood, K.A. Peterson, Commonpath interferometer for frequency-domain optical coherence tomography. Appl. Opt. 42(34), 6953-6958 (2003)

32. M. Born, E. Wolf, Principles of Optics: Electromagnetic Theory of Propagation, Interference and Diffraction of Light (Elsevier, 2013)

33. R.W. Gerchberg, W.O. Saxton, A practical algorithm for the determination of the phase from image and diffraction plane pictures. Optik. 35, 237 (1972)

34. J.R. Fienup, Reconstruction of an object from the modulus of its Fourier transform. Opt. Lett. 3(1), 27-29 (1978)

35. C.U.S. Larsson, A. Beutler, O. Björneholm, F. Federmann, U. Hahn, A. Rieck et al., First results from the high resolution XUV undulator beamline BW3 at HASYLAB. Nucl. Instrum. Methods Phys. Res. Sect. A 337(2), 603-608 (1994)

36. G. Schiwietz, M. Beye, T. Kachel, UE1 $12{ }_{P} G M-1$ : An open-port low-energy beamline at the BESSY II undulator UE112. J. Largescale Res. Facilities 11, 1 (2015)

37. M. Lewenstein, P. Balcou, M.Y. Ivanov, A. L'Huillier, P.B. Corkum, Theory of high-harmonic generation by low-frequency laser fields. Phys. Rev. A 49, 2117-2132 (1994)

38. M. Ferray, A. L'Huillier, X.F. Li, L.A. Lompre, G. Mainfray, C. Manus, Multiple-harmonic conversion of 1064-nm radiation in rare-gase. J. Phys. B. 21(3), L31-L35 (1988)

39. P.W. Wachulak, A. Bartnik, H. Fiedorowicz, P. Rudawski, R. Jarocki, J. Kostecki et al., "Water window" compact, table-top laser plasma soft X-ray sources based on a gas puff target. Nucl. Instrum. Methods Phys. Res. B. 268(10), 1692 (2010)

40. A. Bartnik, H. Fiedorowicz, P. Wachulak, Spectral investigations of photoionized plasmas induced in atomic and molecular gases using nanosecond extreme ultraviolet (EUV) pulses. Phys. Plasmas 21(7), 073303 (2014)

41. P. Wachulak, M. Duda, A. Bartnik, L. Wegrzynski, T. Fok, H. Fiedorowicz, NEXAFS at nitrogen K-edge and titanium L-edge using a laser-plasma soft $\mathrm{x}$-ray source based on a double-stream gas puff target. APL Photonics. 4(3), 030807 (2019)
42. P.W. Wachulak, A. Torrisi, W. Krauze, A. Bartnik, J. Kostecki, M. Maisano et al., A "water window" tomography based on a laserplasma double-stream gas-puff target soft X-ray source. Appl. Phys. B. 125(5), 70 (2019)

43. J. Viefhaus, F. Scholz, S. Deinert, L. Glaser, M. Ilchen, J. Seltmann et al., The variable polarization XUV beamline P04 at PETRA III: optics, mechanics and their performance. Nucl. Instrum. Methods Phys. Res. B. 710, 151 (2013)

44. T. Popmintchev, M.C. Chen, D. Popmintchev, P. Arpin, S. Brown, S. Ališauskas et al., Bright coherent ultrahigh harmonics in the $\mathrm{keV}$ X-ray Regime from mid-infrared femtosecond lasers. Science 336(6086), 1287-1291 (2012)

45. Y. Shamir, J. Rothhardt, J. Hädrich, S. Demmler, M. Tschernajev, J. Limpert et al., High average power $2 \mu \mathrm{m}$ few-cycle optical parametric chirped pulse amplifier at $100-\mathrm{kHz}$ repetition rate. Opt. Lett. 40, 5546 (2015)

46. J. Rothhardt, S. Hädrich, Y. Shamir, M. Tschnernajew, R. Klas, A. Hoffmann et al., High-repetition-rate and high-photon-flux $70 \mathrm{eV}$ high-harmonic source for coincidence ion imaging of gas-phase molecules. Opt. Express 24, 18133 (2016)

47. F. Frassetto, A. Trabattoni, S. Anumula, G. Sansone, F. Calegari, M. Nisoli et al., High-throughput beamline for attosecond pulses based on toroidal mirrors with microfocusing capabilities. Rev. Sci. Instr. 85(10), 103115 (2014)

48. D.J. Merthe, K.A. Goldberg, V.V. Yashchuk, S. Yuan, W.R. McKinney, R. Celestre, An experimental apparatus for diffractionlimited soft X-ray nano-focusing. Proc. SPIE Adv. X-Ray/EUV Opt. Comp. VI 8139, 813907 (2011)

49. Y.X. Wang, W.B. Yun, C. Jacobsen, Achromatic Fresnel optics for wideband extreme-ultraviolet and X-ray imaging. Nature 424(6944), 50-53 (2003)

50. G.J. Stein, P.D. Keathley, P. Krogen, H. Liang, J..P. Siqueira, C..L. Chang, Water-Window Soft $\mathrm{x}$-Ray High-Harmonic Generation up to the Nitrogen K-Edge Driven by a KHz, $2.1 \mu$ m OPCPA Source (J Phys B, 2016)

Publisher's Note Springer Nature remains neutral with regard to jurisdictional claims in published maps and institutional affiliations. 